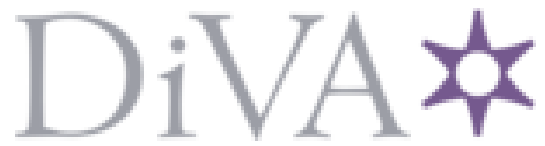

http://www.diva-portal.org

Preprint

This is the submitted version of a paper published in Studies in Computational Intelligence.

Citation for the original published paper (version of record):

Mashad Nemati, H., Fan, Y., Alonso-Fernandez, F. (2016)

Hand Detection and Gesture Recognition Using Symmetric Patterns

Studies in Computational Intelligence, 642: 365-375

https://doi.org/10.1007/978-3-319-31277-4_32

Access to the published version may require subscription.

N.B. When citing this work, cite the original published paper.

Permanent link to this version:

http://urn.kb.se/resolve?urn=urn:nbn:se:hh:diva-40624 


\title{
Hand Detection and Gesture Recognition Using Symmetric Patterns
}

\author{
Hassan Nemati, Yuantao Fan and Fernando Alonso-Fernandez \\ Center for Applied Intelligent Systems Research (CAISR) \\ Halmstad University, Sweden \\ Email: $\{$ Hassan.Nemati, Yuantao.Fan, feralo\}@hh.se
}

\begin{abstract}
Hand detection and gesture recognition is one of the challenging issues in human-robot interaction. In this paper we proposed a novel method to detect human hands and recognize gestures from video stream by utilizing a family of symmetric patterns: log-spiral codes. In this case, several log-family spirals mounted on a hand glove were extracted and utilized for positioning the palm and fingers. The proposed method can be applied in real time and even on a low quality camera stream. The experiments are implemented in different conditions to evaluate the illumination, scale, and rotation invariance of the proposed method. The results show that using the proposed technique we can have a precise and reliable detection and tracking of the hand and fingers with accuracy about $98 \%$.
\end{abstract}

Keywords-Hand Detection, Gesture Recognition, Symmetric Patterns, Log-spiral Codes, Human-Robot Interaction

\section{INTRODUCTION}

Hand detection and gesture recognition is a promising application for human-machine interaction purposes. In this paper we proposed a method using a family of symmetric patterns as features for detecting human hand as well as recognizing several gestures. Utilizing features for identifying position of the object is well-established and widely used paradigm in computer vision and pattern recognition. A number of popular features has been proposed and developed in previous decades for general purpose tasks such as Harris detector [1] and local scaleinvariant features [2], there can be used for hand detection and feature recognition as well. Several other research projects e.g. [3], [4] propose to use human skin as visual features for hand detection.

A hand gesture detector can assist human interaction with machines under various circumstances. For example: (a) operators in factory that has constraints in using other ways of interfacing the machine; (b) disabled or elderly people can interact with machines using natural and universal patterns of motion; (c) for authentication purposes, a person just need to pose the hand in front of a camera and make a series of gestures; (d) provide various ways for interfacing with embedded devices with only camera sensors etc.

Some of the applications such as industrial machine operating require precision and high reliability in tracking the hand. Therefore, unique, robust features must be introduced for this purpose. The work [5] suggests the use of symmetric patterns and corresponding filters for pattern recognition which is later explored by Karlsson and Bigun in [6], where it is described a method to use log-spiral codes as feature for visual positioning. Logspirals are invariant to rotation, scale and drastic changes in intensity. Therefore they are suitable for the type of application that need precision and high reliability. In this paper, we proposed to attach spirals on hand gloves and exploit them as features for both hand detection and gesture recognition.

The main contributions of the paper are the use of Log-spiral codes for hand detection and simple gesture recognition, the use of a spiral pair to make the method more adaptive and robust to changes, the implementation of a way to reject false detection of spirals as well as the analysis of testing the performance of the method under different conditions.

\section{RELATED WORK}

State-of-art approaches for hand detection essentially propose segmentation methods based on features such as skin color, texture, shape and edge detection, e.g. [3] utilize features including shape, context and skin color as multiple proposals for detecting hand and approach described in [4] proposed a way of using color based method to detect hand and fingers for user interaction purpose. Several color-based methods employ artificial markers as features, e.g. [7] utilize a multi-colored glove for hand tracking and method described in [8] uses several types of color markers, attached to fingers, for hand gesture interaction with tabletop. Some other method [9] also take information of background into account for detection. The work in [10] described a method based on Viola-Jones [11] object detection frame work, utilize a hierarchical detection for estimating the positions.

Gesture recognition method were based on the feature extracted from the detection method. One common approach is to use machine learning method. Works [12], [13], [14] utilize Hidden Markov Model to exploit temporal information to recognize hand gesture. Works [15], [16] use Multilayer Perceptron and [17], [18] employ Support Vector Machine to recognize gestures. Those method are conducted in supervised manner but unsupervised methods can be exploited in some cases.

\section{METHOD}

This section describes the algorithm employed for hand detection and gesture recognition. We use symmetry features for such tasks. Symmetry features enable the description of symmetric patterns such as lines, circles, 
parabolas, and so on (Figure 1). These features are extracted via symmetry filters, Equation 1, which output how much of a certain symmetry exist in a local image neighborhood [19], [20]. Concretely, we employ the logspiral family (first family of Figure 1). The exact patterns employed are shown in Figure 2, which are attached to a glove as depicted in Figure 3.

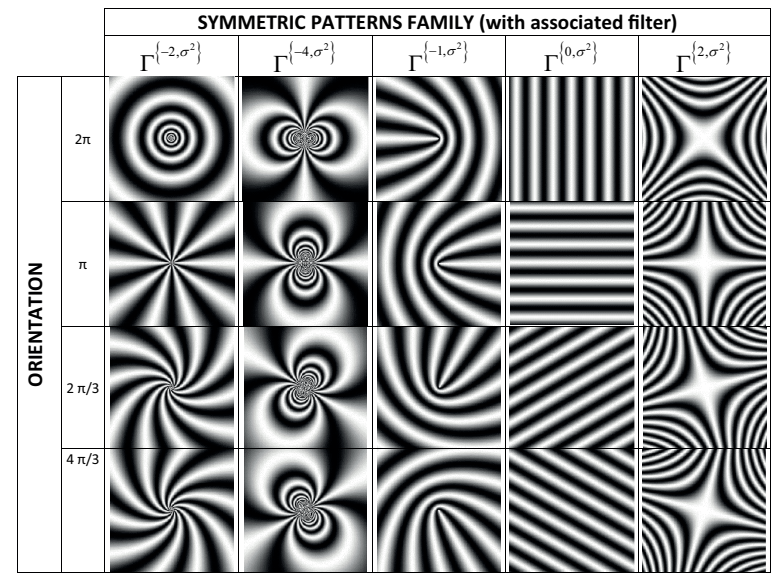

Fig. 1. Example of symmetric patterns. Each column represents one family of patterns differing only by their orientation (given in column 2). The associated filter suitable to detect each family (Equation 2) is also indicated in row 2 .

\section{A. Symmetry filters}

Symmetry filters are a family of filters computed from symmetry derivatives of Gaussians. The $n^{t h}$ symmetry derivative of a Gaussian, $\Gamma^{\left\{n, \sigma^{2}\right\}}$, is obtained by applying the partial derivative operator $D_{x}+i D_{y}=(\partial / \partial x)+$ $i(\partial / \partial y)$, called $1^{\text {st }}$ symmetry derivative, to a Gaussian:

$$
\Gamma^{\left\{n, \sigma^{2}\right\}}=\left\{\begin{array}{cc}
\left(D_{x}+i D_{y}\right)^{n} g(x, y) & (n \geq 0) \\
\left(D_{x}-i D_{y}\right)^{|n|} g(x, y) & (n<0)
\end{array}\right.
$$

Since $D_{x}+i D_{y}$ and $\left(-\frac{1}{\sigma^{2}}\right)(x+i y)$ behave identically when acting on a Gaussian [19], [20], Equation 1 can be rewritten as

$$
\Gamma^{\left\{n, \sigma^{2}\right\}}=\left\{\begin{array}{cc}
\left(-\frac{1}{\sigma^{2}}\right)^{n}(x+i y)^{n} g(x, y) & (n \geq 0) \\
\left(-\frac{1}{\sigma^{2}}\right)^{|n|}(x-i y)^{|n|} g(x, y) & (n<0)
\end{array}\right.
$$

The interest is that these symmetry derivatives of Gaussians are able to detect patterns as those of Figure 1 through the computation of the second order complex moment of the power spectrum via $I_{20}=\left\langle\Gamma^{\left\{n, \sigma_{2}^{2}\right\}}, h\right\rangle$, where $h$ is the complex-valued orientation tensor field given by $h=\left\langle\Gamma^{\left\{1, \sigma_{1}^{2}\right\}}, f\right\rangle^{2}$ and $f$ is the image under analysis [20]. Parameter $\sigma_{1}$ defines the size of the derivation filters used in the computation of $h$, whereas $\sigma_{2}$, used in the computation of $I_{20}$, defines the size extension of the sought pattern.

For each family of symmetric patterns, there is a symmetry filter $\Gamma^{\left\{n, \sigma^{2}\right\}}$ (indexed by $n$ ) suitable to detect the whole family [21]. Figure 1 indicates the filters that are used to detect each family. The local maxima in $\left|I_{20}\right|$ gives the location, whereas the argument of $I_{20}$ at maxima locations gives the group orientation of the detected pattern (except for the first family in Figure 1, $n=-2$, where the 'orientation' represents the chirality of the curves). Therefore, $I_{20}$ encodes how much of a certain type of symmetry exists in a local neighborhood of the image $f$. In addition, a single symmetry filter $\Gamma^{\left\{n, \sigma^{2}\right\}}$ is used for the recognition of the entire family of patterns, regardless of their orientation (or chirality). Symmetry filters have been successfully applied to a wide range of detection tasks such as cross-markers in vehicle crash tests [22], core-points and minutiae in fingerprints [23], [24], or iris boundaries [25]. The beauty of this method is even more emphasized by the fact that $I_{20}$ is computed by filtering in Cartesian coordinates without the need of transformation to curvilinear coordinates (which is implicitly encoded in the filter).

\section{B. Hand detection using log-spirals}

In this work, we employ the set of spirals of order $n=-2$. These are called log-spirals since they are generated by a $\log (z)$ coordinate transformation of 2Dsinusoids $\exp \left(i\left(\omega_{x} x+\omega_{y} y\right)\right)$ [6]. In this case, as introduced above, 'orientation' describes the twist-angle or chirality of the spirals (left/right handedness). Due to the separability property of 2D Gaussian, the detection filter can be re-written as:

$$
\Gamma^{\left\{-2, \sigma^{2}\right\}}=\left(-\frac{1}{\sigma^{2}}\right)^{2}(x-i y)^{2} g(x) g(y)
$$

so the $2 \mathrm{D}$ convolutions can be computed by $1 \mathrm{D}$ convolutions. Moreover, in computing $h, 1 \mathrm{D}$ convolutions can be used as well due to the same property. This results a considerable higher speed, allowing real-time detection. Another advantage of the proposed system is that it does not need training.

In our experiments, we employ 4 different spirals with twist angles $\frac{\pi}{5}, \frac{3 \pi}{5}, \frac{7 \pi}{5}, \frac{9 \pi}{5}$ as shown in Figure 2. They are generated according to the description given in [6], with $L_{0}=12$ legs. These spirals are mounted at the following positions on a glove, as shown in Figure 3:

- One unique spiral on bottom left of the palm

- One unique spiral on bottom right of the palm

- One unique spiral on thumb

- Four same spirals on point finger, middle finger, ring finger, and small finger

Therefore the glove contains 7 spirals which define the hand border. The relative position of these points, the number of detected or not detected points, and the angle of the two spiral at the bottom of the palm are used for hand detection and gesture recognition.

\section{EXPERIMENTAL RESULTS}

In this work we use an ordinary video webcam with resolution of $640 * 480$ and maximum frame rate of 24 . The tests are performed in an offline situation, where 17 different videos containing 22168 frames are recorded 

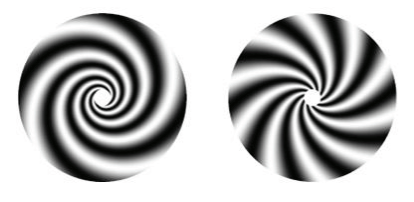

Fig. 2. Spirals employed in our experiments
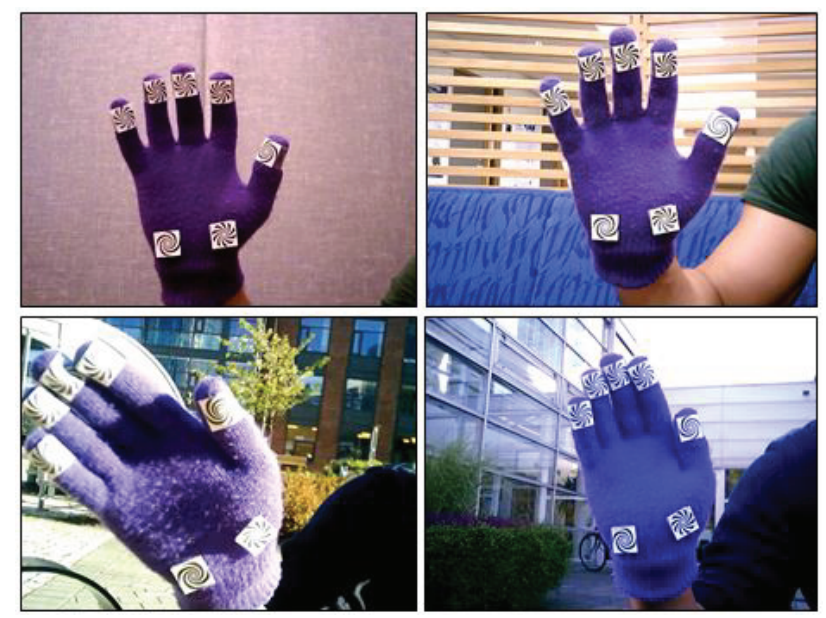

Fig. 3. Example of different test conditions: indoor with simple background (top left), indoor with complex background (top right), in/outdoor with high illumination (bottom left), and outdoor (bottom right)

in different scenarios and then the proposed method is applied on these recorded videos. It takes approximately 0.056 seconds to process a frame on a Macbook that runs OSX 10.9.5 and has an Intel core i7 2.3Ghz processor, which means the method can be applied in real time videos. The distance between the hand and the camera at the first frame is within $10 \mathrm{~cm}$ to $20 \mathrm{~cm}$; this distance may change in the rest of the frames.

\section{A. Hand Detection}

The performance of the proposed method is evaluated in four different conditions: indoor with simple background, indoor with complex background, indoor or outdoor with high illumination, and outdoor. An example of these conditions are shown in Figure 3. In addition to the environmental condition and effects of illumination, we test the detection technique while the hand is rotating in to left or right and also moving forward or backward to the camera (change of scale). These experiments are implemented to test that the proposed technique is robust to changes in illumiation, scale and rotation.

In order to have a scale invariant detection we need to adjust the size of the filter based on the distance between hand and camera. In this case, when the hand move towards the camera the size of the filter should increase and when it move farther away from the camera the filter size should decrease. This can be done by defining the size of the filter not as a fixed value but as a function of distance between hand and camera. To be able to detect this distance, the position of the two spirals at the bottom palm of the hand is considered as the ground truth. Therefore, when in a video frame this length become smaller than a certain value means the hand become farther from the camera and when the length is greater than a certain value means the hand become closer. Using this analogy we are able to modify and update the size of filter depending on the distance between hand and camera and improve the spiral detection algorithm.

In order to evaluate the performance of the proposed method, we used two ways to measure error. In the first way, we count the number of undetected spirals as false negative and then sum the number of these false negatives in all the frames. The expected number of spiral at these experiments is 7 , i.e. all the spirals in all the frames are visible. Consequently, from these 7 spiral if we can detect any of them it will be considered as true positives. The expected number of true positives can be calculated by multiplying the number of frames by 7 (the number of spirals). Therefore, the ratio of the sum of the false negatives over the expected number of true positives gives us the false negative ratio error in the proposed method.

In the second way, we count the number of imperfect frames. In this case, the imperfect frame is defined as the frame that there is exist at least one spiral which is not detected by the method. The ratio of the sum of the imperfect frames over the total number of frames is used as the second way to estimate the performance of the proposed method.

The result of the performance evaluation is shown in Table I. As can be seen in this table, the ratio of error in detecting the spirals in all the tests is less than $2 \%$, i.e. the proposed technique can detect the spirals (the border of the hand) with accuracy about $98 \%$. Furthermore, in more than $92 \%$ of all the test frames the proposed techniques can detect all the spirals without any imperfect frame.

Note that in the high illumination test, when we had direct sun light over the spirals (see Figure 4), the method can not detect all the spirals correctly. In fact, strong sunlight on printed spirals causes reflection with high intensity and consequently increases the imperfect frame ratio error up to 0.082 (see Table I).

A sample scan of the detected spirals from different experiments are shown in Figure 5. In these figures the frame number, filter size, angle of the hand, estimated center of the hand, and the number of true positives in the

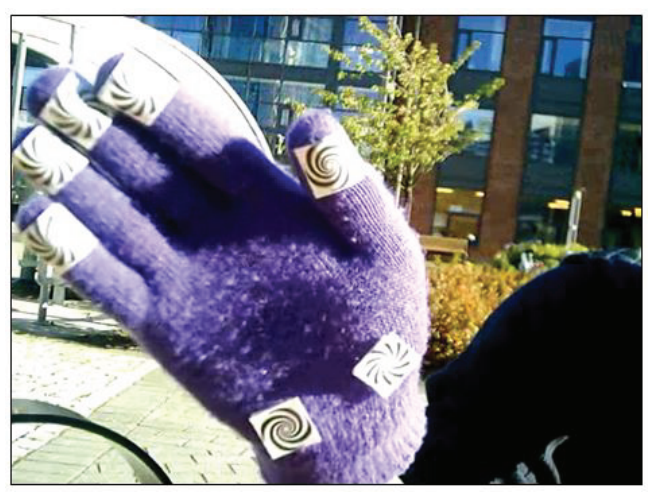

Fig. 4. Effect of direct sun light over the spirals 
TABLE I

THE RESULT OF THE PERFORMANCE EVALUATION - THE RATIO OF ERROR IN DETECTING THE SPIRALS IN ALL THE TESTS IS LESS THAN $2 \%$, THE IMPERFECT FRAME RATIO ERROR IN ALL THE TESTS IS LESS THAN $8.2 \%$

\begin{tabular}{cccccc}
\hline Test & Number & \multicolumn{2}{c}{ False Negative } & \multicolumn{2}{c}{ Imperfect Frame } \\
Environment & of Frames & Ratio & Percent & Ratio & Percent \\
\hline Indoor simple background & 10801 & 0.0096 & $\sim 1 \%$ & 0.0542 & $\sim 5.5 \%$ \\
Indoor complex background & 3917 & 0.0151 & $\sim 1.6 \%$ & 0.0638 & $\sim 6.4 \%$ \\
In/outdoor high intensity & 2432 & 0.0117 & $\sim 1.2 \%$ & 0.0818 & $\sim 8.2 \%$ \\
Outdoor & 5018 & 0.0060 & $\sim 1 \%$ & 0.0296 & $\sim 3 \%$ \\
\hline
\end{tabular}

current frame are specified. Note that the center and angle of the hand are estimated based on the relative position between the two spiral at the bottom palm.

\section{B. Gesture Recognition}

In this section we describe the result of applying our gesture recognition technique. In this case we have defined eight simple gestures as the commands to be sent to an intelligent robot. These commands are defined as "Move to Right", "Move to Left", "Stop", “Turn", "Do Action 1", "Do Action 2", "Do Action 3", and "Do Action 4". These commands and the corresponding results from the gesture recognition technique are shown in Figure 6.

To recognize a gesture we take advantage of the relative location between each unique spiral. Indeed, each individual spiral specifies an specific part of the hand i.e. fingers, thumb, bottom palm left, and bottom palm right. For example, the angle of the hand can be computed by using the two spirals in the bottom palm. This can be used for recognizing commands such as "Move to Right"and "Move to Left". Combining the information from other spirals and the angle of hand will give us the possibility to recognize other gestures.

At each frame, based on the number and the position of the spirals, a command is generated. In order to avoid falsely recognize random movement of the hand as a command or a gesture from an imperfect frame, the proposed technique waits to receive the same command for at least 12 consecutive frames (half a second). When it became certain about the captured command it will demonstrate the commands on the screen. Using this will avoid sending unexpected commands during changes in the hand

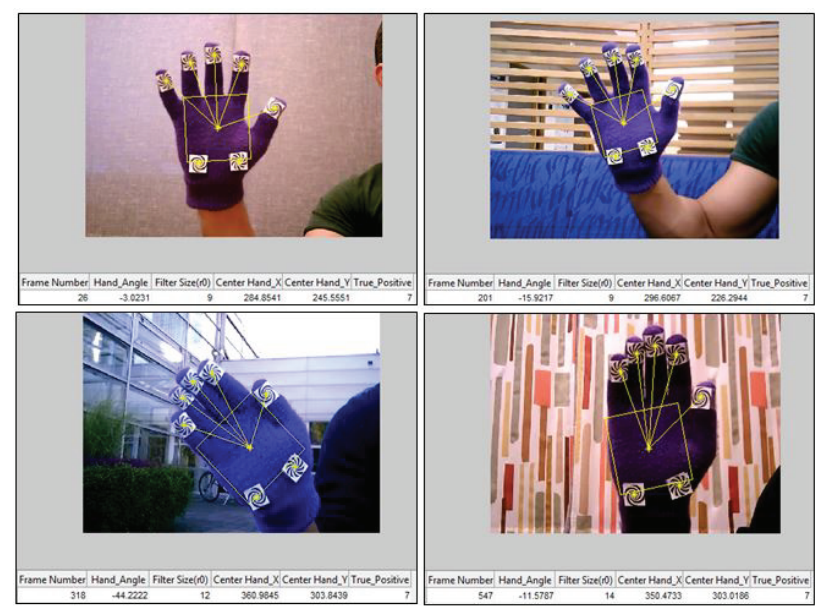

Fig. 5. Sample scans of applying the hand detection technique gestures. Therefore, the proposed technique will be less sensitive to fast changes and receiving hand position from an imperfect frame. Using the proposed hand detection and gesture recognition technique, we are able to detect all the gestures and corresponding commands correctly i.e. all the hand gestures and corresponding commands in all the recorded videos are correctly detected and classified.

\section{CONCLUSION AND DiscUSSION}

In this paper we presented a novel technique for human hand detection using symmetric log-spiral patterns. Four different types of log-spirals are generated and mounted on a hand glove. The relative location of these spirals are used for positioning of the palm, fingers, and the angle of the hand. By taking advantage of the location of each spiral we are able to recognize simple commands from the hand gestures. The experimental results showed that the proposed technique can provide a reliable hand detection and gesture recognition with accuracy about $98 \%$.

To improve the hand detection technique we can use tracking methods such as Kalman Filters and Extended Kalman Filters. In this case, by estimating the position of the fingers in the next frame we can reduce the number of imperfect frames.

Furthermore, we can create a precise dictionary of language signs using a person records the hand gestures with the glove. This can be used to reproduce the messages by an avatar or to create new messages (not recorded) by combining individual signs. With this, we do not need to store videos, but sequences of spiral points which takes less space for storage and for transmission.

\section{ACKNOWLEDGMENTS}

F. A.-F. thanks the Swedish Research Council for funding this research. Authors also acknowledge the CAISR program of the Swedish Knowledge Foundation.

\section{REFERENCES}

[1] C. Harris and M. Stephens, "A combined corner and edge detector." in Alvey vision conference, vol. 15. Citeseer, 1988, p. 50.

[2] D. G. Lowe, "Object recognition from local scale-invariant features," in Computer vision, 1999. The proceedings of the seventh IEEE international conference on, vol. 2. Ieee, 1999, pp. 11501157.

[3] A. Mittal, A. Zisserman, and P. H. Torr, "Hand detection using multiple proposals." in BMVC. Citeseer, 2011, pp. 1-11.

[4] S. K. Kang, M. Y. Nam, and P. K. Rhee, "Color based hand and finger detection technology for user interaction," in Convergence and Hybrid Information Technology, 2008. ICHIT'08. International Conference on. IEEE, 2008, pp. 229-236.

[5] D. Teferi and J. Bigun, "Multi-view and multi-scale recognition of symmetric patterns," in Image analysis. Springer, 2009, pp. 657-666.

[6] S. Karlsson and J. Bigun, "Synthesis and detection of log-spiral codes," in SSBA Symposium $i$ bildanalys, 17-18 mars 2011, Linköping, 2011, p. 4. 

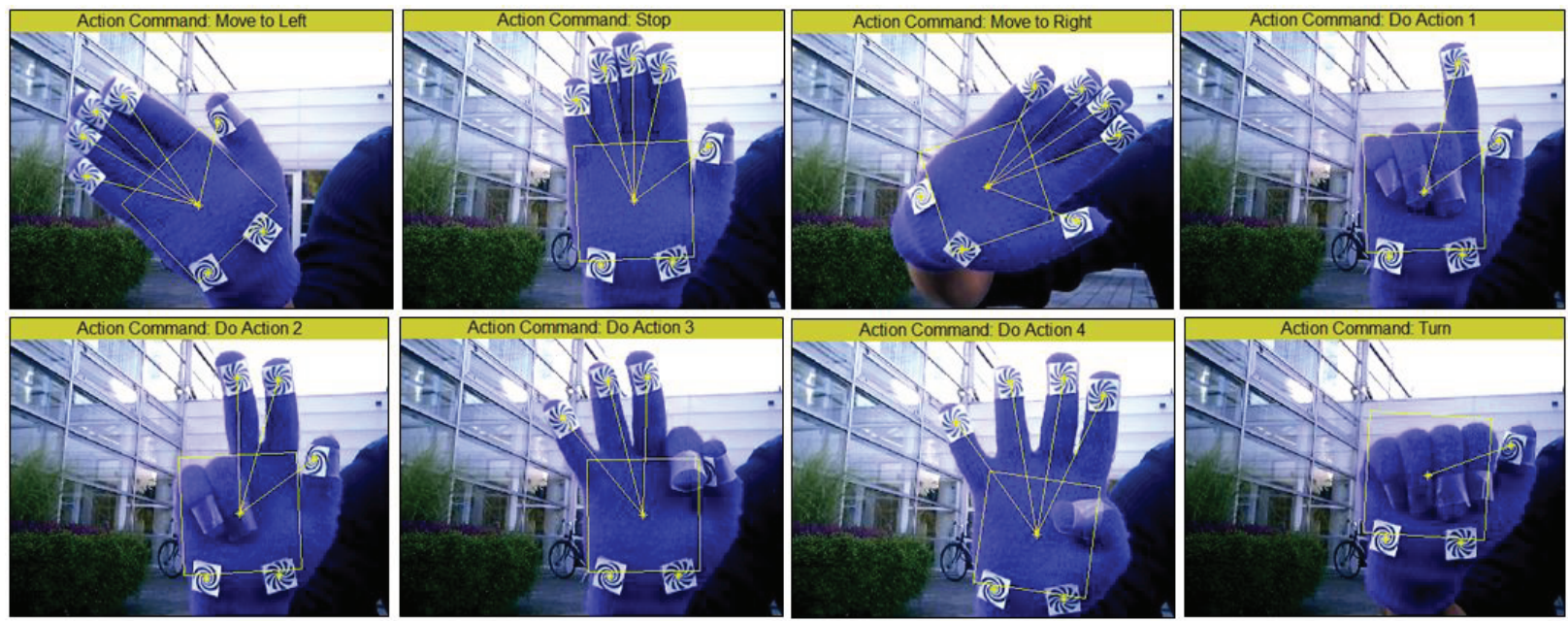

Fig. 6. Simple commands used for hand gesture detection

[7] R. Y. Wang and J. Popović, "Real-time hand-tracking with a color glove," ACM Transactions on Graphics (TOG), vol. 28, no. 3, p. 63,2009 .

[8] A. Bellarbi, S. Benbelkacem, N. Zenati-Henda, and M. Belhocine, "Hand gesture interaction using color-based method for tabletop interfaces," in Intelligent Signal Processing (WISP), 7th International Symposium on. IEEE, 2011, pp. 1-6.

[9] X. Zhu, J. Yang, and A. Waibel, "Segmenting hands of arbitrary color," in Automatic Face and Gesture Recognition, 2000. Proceedings. Fourth IEEE International Conference on. IEEE, 2000, pp. 446-453.

[10] B. Stenger, A. Thayananthan, P. H. Torr, and R. Cipolla, "Hand pose estimation using hierarchical detection," in Computer Vision in Human-Computer Interaction. Springer, 2004, pp. 105-116.

[11] P. Viola, M. J. Jones, and D. Snow, "Detecting pedestrians using patterns of motion and appearance," International Journal of Computer Vision, vol. 63, no. 2, pp. 153-161, 2005.

[12] Z. Yang, Y. Li, W. Chen, and Y. Zheng, "Dynamic hand gesture recognition using hidden markov models," in Computer Science \& Education (ICCSE), 2012 7th International Conference on. IEEE, 2012, pp. 360-365.

[13] B. Kohn, A. N. Belbachir, and A. Nowakowska, "Real-time gesture recognition using bio inspired 3d vision sensor," in Computer Vision and Pattern Recognition Workshops (CVPRW), 2012 IEEE Computer Society Conference on. IEEE, 2012, pp. 37-42.

[14] F.-S. Chen, C.-M. Fu, and C.-L. Huang, "Hand gesture recognition using a real-time tracking method and hidden markov models," Image and vision computing, vol. 21, no. 8, pp. 745-758, 2003.

[15] R.-D. Vatavu, Ş.-G. Pentiuc, C. Chaillou, L. Grisoni, and S. Degrande, "Visual recognition of hand postures for interacting with virtual environments," Advances in Electrical and Computer Engineering, vol. 6, no. 13, pp. 55-58, 2006.

[16] S. G. Wysoski, "A rotation invariant static hand gesture recognition system using boundary information and neural networks," $\mathrm{Ph}$.D. dissertation, NAGOYA INSTITUTE OF TECHNOLOGY, 2003.

[17] A. Sultana and T. Rajapuspha, "Vision based gesture recognition for alphabetical hand gestures using the svm classifier," International Journal of Computer Science and Engineering Technology, vol. 3, no. 7, 2012.

[18] A. Pradhan, M. Ghose, M. Pradhan, S. Qazi, T. Moors, I. M. E. EL-Arab, H. S. El-Din, H. A. Mohamed, U. Syed, A. Memon et al., "A hand gesture recognition using feature extraction," Int $J$ Curr Eng Technol, vol. 2, no. 4, pp. 323-327, 2012.

[19] J. Bigun, T. Bigun, and K. Nilsson, "Recognition by symmetry derivatives and the generalized structure tensor," Pattern Analysis and Machine Intelligence, IEEE Transactions on, vol. 26, no. 12, pp. 1590-1605, 2004.

[20] J. Bigun, Vision with direction. Springer, 2006.

[21] J. Bigün, "Pattern recognition in images by symmetries and coordinate transformations," Computer Vision and Image Understanding, vol. 68, no. 3, pp. 290-307, 1997.

[22] J. Bigün, G. H. Granlund, and J. Wiklund, "Multidimensional orientation estimation with applications to texture analysis and optical flow," IEEE Transactions on Pattern Analysis \& Machine Intelligence, no. 8, pp. 775-790, 1991.

[23] K. Nilsson and J. Bigun, "Localization of corresponding point in fingerprints by complex filtering," Pattern Recognition Letters, vol. 24, no. 13, pp. 2135-2144, 2003.

[24] H. Fronthaler, K. Kollreider, J. Bigun, J. Fierrez, F. AlonsoFernandez, J. Ortega-Garcia, and J. Gonzalez-Rodriguez, "Fingerprint image-quality estimation and its application to multialgorithm verification," Information Forensics and Security, IEEE Transactions on, vol. 3, no. 2, pp. 331-338, 2008.

[25] F. Alonso-Fernandez and J. Bigun, "Iris boundaries segmentation using the generalized structure tensor. a study on the effects of image degradation," in Biometrics: Theory, Applications and Systems (BTAS), 2012 IEEE Fifth International Conference on. IEEE, 2012, pp. 426-431. 